\title{
Trustworthy Machine Learning: Fairness and Robustness
}

\author{
Haochen Liu \\ Data Science and Engineering Lab \\ Michigan State University \\ liuhaoc1@msu.edu
}

\begin{abstract}
In recent years, machine learning (ML) technologies have experienced swift developments and attracted extensive attention from both academia and industry. The applications of ML are extended to multiple domains, from computer vision, text processing, to recommendations, etc. However, recent studies have uncovered the untrustworthy side of ML applications. For example, ML algorithms could show human-like discrimination against certain individuals or groups, or make unreliable decisions in safety-critical scenarios, which implies the absence of fairness and robustness, respectively. Consequently, building trustworthy machine learning systems has become an urgent need. My research strives to help meet this demand. In particular, my research focuses on designing trustworthy ML models and spans across three main areas: (1) fairness in ML, where we aim to detect, eliminate bias and ensure fairness in various ML applications; (2) robustness in ML, where we seek to ensure the robustness of certain ML applications towards adversarial attacks; (3) specific applications of ML, where my research involves the development of ML-based natural language processing (NLP) models and recommendation systems.
\end{abstract}

\section{ACM Reference Format:}

Haochen Liu. 2022. Trustworthy Machine Learning: Fairness and Robustness. In Proceedings of the Fifteenth ACM International Conference on Web Search and Data Mining (WSDM '22), February 21-25, 2022, Tempe, AZ, USA. ACM, New York, NY, USA, 2 pages. https://doi.org/10.1145/3488560.3502211

\section{INTRODUCTION}

Along with the rapid and impressive development of ML algorithms, their untrustworthy sides have also been exposed. For example, ML systems can cause bias and unfairness. ML-based chatbots trained on human conversation data could produce indecent, racist, and sexist content [22] that offends users and has a negative social impact. In addition, safety-critical ML systems are shown to be vulnerable to adversarial attacks. Deep image recognition systems in autonomous vehicles could fail to distinguish road signs modified by malicious attackers [23]. Such vulnerabilities can cause severe social issues and significant economic loss, and hereby prevent ML from further advancement and wider application.

Consequently, the concept of "trustworthy machine learning" [21] is proposed, which requires an ML system to meet the requirements of several dimensions to be trustworthy for humans [15]. The two most important dimensions are fairness and robustness.

Permission to make digital or hard copies of part or all of this work for personal or classroom use is granted without fee provided that copies are not made or distributed for profit or commercial advantage and that copies bear this notice and the full citation on the first page. Copyrights for third-party components of this work must be honored

For all other uses, contact the owner/author(s).

WSDM '22, February 21-25, 2022, Tempe, AZ, USA

(C) 2022 Copyright held by the owner/author(s)

ACM ISBN 978-1-4503-9132-0/22/02.

https://doi.org/10.1145/3488560.3502211
The fairness requires an ML model to avoid discriminatory behaviors in human-machine interactions and ensure fairness in decision making for any individuals or groups. The robustness requires an ML system to be robust to the noisy perturbations of inputs and to be able to make secure decisions. My research focuses on trustworthy ML, and specifically involves three problems: (1) fairness in ML, (2) robustness in ML, and (3) specific ML applications. In particular, my research on the above three problems mainly focuses on the applications of ML in two fields: NLP and recommendation systems.

\section{RELATED WORK}

Fairness in Machine Learning. Increasing attempts have been made to detect and mitigate bias in ML models on various tasks. Initially, studies investigate how algorithms can be biased in classification tasks [5]. Later, in the domain of computer vision, researchers show that ML-based face recognition [3] and object detection [19] models perform unfairly for different demographic groups. Besides, a lot of works examine the bias in language-related tasks, including word embedding [2], machine translation [18], etc.

Robustness in Machine Learning. In recent years, researchers have become aware of the fact that although ML systems can perform well when in a perfect and stable environment, when placed in the real world, they are quite easily susceptible to being attacked. The work [20] first investigates the vulnerability of DNN-based image classifiers by crafting adversarial examples with imperceptible perturbations that lead the classifier to make mistakes. Attacking NLP models are more challenging since the inputs are discrete texts instead of continuous values such as image inputs [24]. The robustness problems in text classification [6], machine translation [1], and reading comprehension [4], etc., have been investigated.

\section{OVERVIEW OF MY RESEARCH}

While there are a growing number of works related to trustworthy ML, the study on fairness issues and robustness issues of certain ML tasks is still absent or in the early stages. In this section, I will present my research works.

\subsection{Fairness in Machine Learning}

Detecting and Mitigating Bias in Dialogue Models. As one crucial ML application, dialogue systems have been extensively applied in our society. They are usually built with real human conversational data; thus they could inherit some bias issues which are held in the real world. However, the fairness of dialogue systems had not been well investigated. In [8] and [14], we perform a pioneering study about the fairness issues in dialogue systems. In particular, we propose a standard definition of fairness in dialogue systems; we construct a benchmark dataset; and we propose quantitative measures to understand fairness in dialogue models. Finally, 
we propose a novel framework based on adversarial learning to mitigate gender bias in dialogue systems.

Understanding and Alleviating Implicit Bias in Text Classification. In [10], we verify that implicit bias exists in different text classification tasks for different demographic groups, and we build a learning-based interpretation method to deepen our knowledge of implicit bias. Besides, we propose a framework Debiased-TC to train deep text classifiers to make predictions on the right features and consequently mitigate implicit bias.

Handling Annotator Group Bias in Crowdsourcing. Crowdsourcing is a popular method for collecting training data for ML models. In [13], we examine the annotator group bias in real-world crowdsourcing datasets. Then, we propose a novel probabilistic graphical framework to capture the bias during training and develop an extended expectation maximization (EM) training algorithm to address the bias.

Mitigating selection bias in recommendation systems. Recommendation systems are trained on historical rating data explicitly provided by users, which suffer from selection bias. In [12], we propose a novel self-supervised learning (SSL) framework Rating Distribution Calibration (RDC) to alleviate the negative impacts of selection bias on recommendation models.

\subsection{Robustness in Machine learning}

Attacking neural dialogue models. We study whether dialogue models can be manipulated to produce inappropriate responses. In [9] and [16], we investigate whether we can craft input sentences that result in a black-box neural dialogue model having its outputs contain target words or match target sentences. Two reinforcement learning based models are developed to automatically generate such desired inputs.

\subsection{Machine Learning Applications}

Besides the fairness and robustness in ML, I have also conducted research on specific applications in NLP and recommendations. Specifically, in [11], we develop a novel personalized multimodal feedback generation framework, which automatically produces personalized textual feedback to evaluate a video assignment based on its multimodal information (image, audio and text). In [17, 25, 26], we use the AutoML technology to automatically search the embedding sizes and loss functions for recommendation models, which improves their space efficiency and performances.

\section{FUTURE DIRECTIONS}

Language model pre-training is a crucial task in NLP and it has been verified that such language models can exhibit human-like bias [7] Although there are a few works studying the bias issues in language modeling, they only focus on the bias in the language model itself but overlook the impacts of the bias of the pre-training language model on downstream models. I plan to investigate whether downstream NLP models can inherit the bias in pre-training language models and how to prevent the spread of bias. In terms of ML-based recommendations, there are still some possibilities not investigated that we can utilize AutoML to automate the design of recommendation models. I would like to investigate using AutoML to adaptively choose desirable architectures for recommendation models.

\section{ACKNOWLEDGMENTS}

Haochen Liu and his advisor Dr. Jiliang Tang are supported by the National Science Foundation (NSF) under grant numbers IIS1714741, CNS1815636, IIS1845081, IIS1907704, DRL2025244, IIS1928278, IIS1955285, IOS2107215, IOS2035472 and Army Research Office (ARO) under grant number W911NF-21-1-0198.

\section{REFERENCES}

[1] Yonatan Belinkov and Yonatan Bisk. 2018. Synthetic and Natural Noise Both Break Neural Machine Translation. In ICLR.

[2] Tolga Bolukbasi, Kai-Wei Chang, James Y Zou, Venkatesh Saligrama, and Adam T Kalai. 2016. Man is to computer programmer as woman is to homemaker? debiasing word embeddings. In NeurIPS.

[3] Joy Buolamwini and Timnit Gebru. 2018. Gender shades: Intersectional accuracy disparities in commercial gender classification. In FAccT.

[4] Alvin Chan, Lei Ma, Felix Juefei-Xu, Xiaofei Xie, Yang Liu, and Yew Soon Ong. 2018. Metamorphic relation based adversarial attacks on differentiable neural computer. arXiv preprint (2018).

[5] Faisal Kamiran and Toon Calders. 2009. Classifying without discriminating. In International Conference on Computer, Control and Communication.

[6] Qi Lei, Lingfei Wu, Pin-Yu Chen, Alexandros G Dimakis, Inderjit S Dhillon, and Michael Witbrock. 2018. Discrete attacks and submodular optimization with applications to text classification. arXiv preprint (2018).

[7] Paul Pu Liang, Chiyu Wu, Louis-Philippe Morency, and Ruslan Salakhutdinov. 2021. Towards understanding and mitigating social biases in language models. In ICML.

[8] Haochen Liu, Jamell Dacon, Wenqi Fan, Hui Liu, Zitao Liu, and Jiliang Tang. 2020. Does Gender Matter? Towards Fairness in Dialogue Systems. In COLING.

[9] Haochen Liu, Tyler Derr, Zitao Liu, and Jiliang Tang. 2019. Say what i want: Towards the dark side of neural dialogue models. arXiv preprint (2019).

[10] Haochen Liu, Wei Jin, Hamid Karimi, Zitao Liu, and Jiliang Tang. 2021. The Authors Matter: Understanding and Mitigating Implicit Bias in Deep Text Classification. In Findings of ACL-IFCNLP.

[11] Haochen Liu, Zitao Liu, Zhongqin Wu, and Jiliang Tang. 2020. Personalized Multimodal Feedback Generation in Education. In COLING.

[12] Haochen Liu, Da Tang, Ji Yang, Xiangyu Zhao, Jiliang Tang, and Youlong Cheng. 2021. Self-supervised Learning for Alleviating Selection Bias in Recommendation Systems. (2021).

[13] Haochen Liu, Joseph Thekinen, Sinem Mollaoglu, Da Tang, Ji Yang, Youlong Cheng, Hui Liu, and Jiliang Tang. 2021. Toward Annotator Group Bias in Crowdsourcing. arXiv preprint (2021).

[14] Haochen Liu, Wentao Wang, Yiqi Wang, Hui Liu, Zitao Liu, and Jiliang Tang. 2020. Mitigating Gender Bias for Neural Dialogue Generation with Adversarial Learning. In $E M N L P$.

[15] Haochen Liu, Yiqi Wang, Wenqi Fan, Xiaorui Liu, Yaxin Li, Shaili Jain, Anil K Jain, and Jiliang Tang. 2021. Trustworthy ai: A computational perspective. arXiv preprint (2021)

[16] Haochen Liu, Zhiwei Wang, Tyler Derr, and Jiliang Tang. 2020. Chat as expected: Learning to manipulate black-box neural dialogue models. arXiv preprint (2020).

[17] Haochen Liu, Xiangyu Zhao, Chong Wang, Xiaobing Liu, and Jiliang Tang. 2020. Automated embedding size search in deep recommender systems. In SIGIR.

[18] Marcelo OR Prates, Pedro H Avelar, and Luis C Lamb. 2019. Assessing gender bias in machine translation: a case study with google translate. Neural Computing and Applications (2019)

[19] Hee Jung Ryu, Margaret Mitchell, and Hartwig Adam. 2017. Improving smiling detection with race and gender diversity. arXiv preprint (2017).

[20] Christian Szegedy, Wojciech Zaremba, Ilya Sutskever, Joan Bruna, Dumitru Erhan, Ian Goodfellow, and Rob Fergus. 2014. Intriguing properties of neural networks.

[21] Kush R Varshney. 2019. Trustworthy machine learning and artificial intelligence. XRDS: Crossroads, The ACM Magazine for Students (2019).

[22] Marty J Wolf, Keith W Miller, and Frances S Grodzinsky. 2017. Why we should have seen that coming: comments on microsoft's tay "experiment," and wider implications. The ORBIT fournal (2017).

[23] Han Xu, Yao Ma, Hao-Chen Liu, Debayan Deb, Hui Liu, Ji-Liang Tang, and Anil K Jain. 2020. Adversarial attacks and defenses in images, graphs and text: A review. International fournal of Automation and Computing (2020).

[24] Wei Emma Zhang, Quan Z Sheng, Ahoud Abdulrahmn F Alhazmi, and Chenliang Li. 2019. Generating textual adversarial examples for deep learning models: A survey. arXiv preprint (2019).

[25] Xiangyu Zhao, Haochen Liu, Wenqi Fan, Hui Liu, Jiliang Tang, and Chong Wang. 2021. AutoLoss: Automated Loss Function Search in Recommendations. arXiv preprint (2021).

[26] Xiangyu Zhao, Haochen Liu, Hui Liu, Jiliang Tang, Weiwei Guo, Jun Shi, Sida Wang, Huiji Gao, and Bo Long. 2021. AutoDim: Field-aware Embedding Dimension Searchin Recommender Systems. In Web Conference. 